\title{
Erratum: Axion like particles and the inverse seesaw mechanism
}

\author{
C.D.R. Carvajal, ${ }^{a}$ A.G. Dias, ${ }^{a}$ C.C. Nishi ${ }^{b, c}$ and B.L. Sánchez-Vega ${ }^{d}$ \\ ${ }^{a}$ Universidade Federal do ABC, Centro de Ciências Naturais e Humanas, \\ Av. dos Estados, 5001, 09210-580, Santo André, SP, Brasil \\ ${ }^{b}$ Maryland Center for Fundamental Physics, University of Maryland, \\ College Park, MD 20742, U.S.A. \\ ${ }^{c}$ Universidade Federal do ABC, Centro de Matemática, Computação e Cognição, \\ Av. dos Estados, 5001, 09210-580, Santo André, SP, Brasil \\ ${ }^{d}$ Argonne National Laboratory, \\ 9700 S. Cass Avenue, Argonne, Illinois 60439, U.S.A. \\ E-mail: crisdaruiz@gmail.com, alex.dias@ufabc.edu.br, \\ celso.nishi@ufabc.edu.br, brucesanchez@anl.gov
}

ERRATUM TO: JHEP05(2015)069

ARXIV EPRINT: 1503.03502

The two models containing two ALPs in section 2.3 of ref. [1] lead to too light non-SM charged leptons $E$ of only a few GeV. (The single ALP model of section 2.2 does not suffer from this problem.)

This problem can be amended without significant modifications by considering two heavy vector-like fermions $E$ and $E^{\prime}$ instead of one. Instead of the last two terms of eq. (2.40), (2.41) and (2.47) of [1], we should consider the four terms

$$
\begin{aligned}
-\mathscr{L} \supset & k_{i} \frac{\sigma \sigma^{\prime}}{M_{\mathrm{Pl}}^{2}} \overline{L_{i}} H E_{R}+k_{i}^{\prime} \frac{\sigma \sigma^{\prime}}{M_{\mathrm{Pl}}^{2}} \overline{L_{i}} H E_{R}^{\prime} \\
& +k_{E} \sigma \overline{E_{L}} E_{R}+k_{E}^{\prime} \sigma^{\prime} \overline{E_{L}^{\prime}} E_{R}^{\prime}+\text { H.c. },
\end{aligned}
$$

where we already fixed the $r, r^{\prime}$ powers without affecting any formula. One should use $s=s^{\prime}=1$ if needed. Now the heavy leptons $E, E^{\prime}$ have intermediate scale masses of order $10^{9 \div 10} \mathrm{GeV}$. The charge of the discrete symmetries are only modified for $E_{L, R}$ and $E_{L, R}^{\prime}$ in a predictable manner; see table below. Appropriate normalization of these charges leads to the PQ charges $X, X^{\prime}$ and the extended lepton numbers $L, L^{\prime}$. Note that $\mathrm{U}(1)_{X}\left[\mathrm{U}(1)_{X^{\prime}}\right]$ is vectorial for $E^{\prime}(E)$ and that the first two terms of the Lagrangian above implies $E_{R}$ and $E_{R}^{\prime}$ have equal charges $\left(X, X^{\prime}, Z, Z^{\prime}, L, L^{\prime}\right)$. The rest of the formulas and the phenomenological consequences remain unchanged.

Additionally, the lowest order operator coupling $\bar{L}-S_{R}$ for model II is $\bar{L} \tilde{H} S_{R} \sigma^{4} \sigma^{\prime}$ instead of $\bar{L} \tilde{H} S_{R} \sigma^{2} \sigma^{\prime *}$. This implies $m_{\mathrm{DS}}$ is negligible in eq. (2.52). 


\begin{tabular}{|c|c|c|c|c|}
\hline & $E_{L}$ & $E_{R}$ & $E_{L}^{\prime}$ & $E_{R}^{\prime}$ \\
\hline $\mathbb{Z}_{8}$ & 2 & 1 & 1 & 1 \\
\hline $\mathbb{Z}_{11}$ & 1 & 1 & 3 & 1 \\
\hline
\end{tabular}

\begin{tabular}{|c|c|c|c|c|}
\hline & $E_{L}$ & $E_{R}$ & $E_{L}^{\prime}$ & $E_{R}^{\prime}$ \\
\hline $\mathbb{Z}_{8}$ & 3 & 2 & 2 & 2 \\
\hline $\mathbb{Z}_{10}$ & -1 & -1 & 1 & -1 \\
\hline
\end{tabular}

Table 1. Corrected charges for model I (left) and model II (right).

Open Access. This article is distributed under the terms of the Creative Commons Attribution License (CC-BY 4.0), which permits any use, distribution and reproduction in any medium, provided the original author(s) and source are credited.

\section{References}

[1] C.D.R. Carvajal, A. G. Dias, C.C. Nishi and B.L. Sánchez-Vega, Axion like particles and the inverse seesaw mechanism, JHEP 05 (2015) 069 [arXiv:1503.03502] [INSPIRE]. 